\section{The length of the shortest period of rests of numbers $n^{n}$}

\author{
by R. HAMPEL (Warszawa)
}

W. Sierpiński proves ${ }^{1}$ ) that the last figures of the numbers $n^{n}$ form the periodical sequence whose shortest period consists of 20 terms, and that for every natural $m$ the rests $\bmod m$ of the numbers $n^{n}$ $(n=1,2,3, \ldots)$ form an infinite periodical sequence.

My aim in this paper ${ }^{2}$ ) is to calculate the length of the shortest period $\bmod m$.

Let us suppose that

$$
m=\prod_{l=1}^{k} p_{l}^{a_{l}}, \quad \alpha_{l} \geqslant 1, \quad 2 \leqslant p_{i}<p_{i+1} \quad(i=1,2, \ldots, k-1) .
$$

I shall prove that

$$
n^{n} \equiv r_{n}^{(m)}(\bmod m)
$$

the rests $r_{n}^{(m)}$ forming the periodical sequence whose shortest period $S_{m}$ satisfies the relation

$$
S_{m} \mid \varphi(m) \prod_{l=1}^{k} p_{l}
$$

exactly

$$
S_{m}=\left\{p_{i} \varphi\left(p_{i}^{a_{i}}\right)\right\} \quad \text { i.e. } \quad S_{m}=\left\{m ; p_{i}-1\right\} \quad(i=1,2, \ldots, k),
$$
$\varphi(m)$ denotes the well known function of Gauss and \{\} - the least com-
mon multiple of corresponding numbers.

It is obvious that to prove the relation (1) we have to show for $n \geqslant n_{0}$ $\left(n_{0}\right.$ denotes the well determined natural number given below) the validity of the congruence

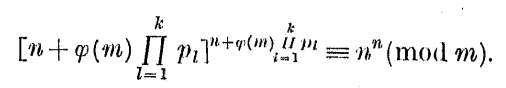

1) W. Sierpiński, Sur la periodicité $\bmod m$ de certaines suites infinies d'entiers, Annales de la Soc. Pol. Math. 23 (1950), p. 256.

${ }^{2}$ ) Communication presented to the Polish Mathematical Society, Section of Warsaw, March 13, 1953,
Considering

we get from (3)

$$
m \mid \varphi(m) \prod_{l=1}^{k} p_{l}=m \prod_{l=1}^{k}\left(p_{l}-1\right)
$$

$u^{n}\left[n^{\varphi(m) I m}{ }_{l=1}^{n}-1\right] \equiv 0(\bmod m)$

$1^{0}(n, m)=1$. On applying the theorem of Euler we immediately see that the congruence (3) is valid.

$2^{\mathfrak{a}}(n, m) \neq 1$. Suppose that

$$
(n, m)=\prod_{t=1}^{r} p_{l_{t}}^{\beta_{l_{4}}}, \quad r \leqslant k, \quad 1 \leqslant \beta_{l_{t}} \leqslant \alpha_{l_{t}}, \quad 1 \leqslant l_{1}<l_{2}<\ldots<l_{r} \leqslant k .
$$

We begin the calculation of the period from the least natural number $n=n_{0}$ which satisfies the inequalities $n \beta_{l_{l}} \geqslant \alpha_{l_{l}}, t=1,2, \ldots, r$. e. from such $n_{0}$ that

$$
n_{0} \geqslant \max \frac{\alpha_{l_{1}}}{\beta_{l_{l}}} \quad \text { and } \quad n_{0}-1<\max \frac{\alpha_{l_{l}}}{\beta_{l_{l}}} \quad(t=1,2, \ldots, v) \text {. }
$$

Applying the symbol $\mathrm{E}$ (entier) we get

$$
n_{0}=-\mathrm{E}\left(-\max \frac{u_{l_{t}}}{\beta_{l_{\iota}}}\right) \text {. }
$$

We shall prove the validity of the congruence (4) for every $n \geqslant n_{0}$. Applying the multiplicativeness of the function $\varphi(m)$ and considering that the two obtained factors are mutually prime we may write this congruence in the form

$$
m^{n}\left(n^{y}-1\right) \equiv 0(\bmod m)
$$

where

$$
\nu=\varphi\left(m / \prod_{t=1}^{r} p_{l_{t}}^{a_{l}}\right) \varphi\left(\prod_{t=1}^{r} p_{l_{t}}^{a_{l_{t}}} \prod_{t=1}^{k} p_{l}\right.
$$

but $n^{n} \equiv 0\left(\bmod \prod_{t=1}^{r} p_{l_{t}}^{a_{t_{l}}}\right) \quad$ because for $n \geqslant n_{0}$

$$
n \beta_{l_{l}} \geqslant u_{l_{l}}
$$

Applying the theorem of Euler to the expression in brackets in (5) and taking into account that

$$
\left(\frac{m}{\prod_{t=1}^{r} p_{l_{t}}^{a_{l_{t}}}} ; n\right)=1
$$

we prove immediately the theorem. 
It is sufficient to take $n=n_{0}=\max a_{i}, i=1,2, \ldots, k$.

Before proving that the shortest period $S_{m}=\left\{m ; p_{i}-1\right\}$ I shall discuss some special cases.

Let us take $m=p^{\alpha}$. According to the relation (1) we have (6)

$$
S_{m} \leqslant p^{a}(p-1)
$$

I shall prove that

$$
S_{m}=p \varphi\left(p^{\alpha}\right)=p^{\alpha}(p-1) .
$$

We distinguish two cases:

$$
1^{0} \quad a=1 \text {, i. e. } m=p, S_{p}=p(p-1) \text {. }
$$

I report the proof of W. Sierpinski given in his letter of 6.5 .1953 .

It was proved above that $S_{p} \mid p(p-1)$. Therefore it is sufficient to show that the period $S<p(p-1)$ does not exist. But for the prime mod $p$ we have $n^{n} \equiv 0(\bmod p)$, if and only if $n \equiv 0(\bmod p)$, which involves $p \mid S$ i. e. $S=p k$ ( $k$ - natural number $)$.

Let us suppose now that $S<p(p-1)$, it means that $k<p-1$, i.e. since $S$ is a period, we may write

$$
(n+S)^{n+s} \equiv n^{n}(\bmod p)
$$

for natural $n$; hence, regarding $S=p k$, it follows that

$$
n^{n} n^{p k} \equiv n^{n}(\bmod p) \text {; }
$$

the last congruence for $(n, p)=1$ gives immediately $n^{p k} \equiv 1(\bmod p)$. But according to the theorem of Fermat we have

$n^{p-1} \equiv 1(\bmod p)$ for $(n, p)=1$, hence $n^{(p-1) k} \equiv 1(\bmod p)$ for $\quad(n, p)=1$.

Since $n^{p k}=n^{(p-1) k} n^{k}$, we obtain

$$
n^{k} \equiv 1(\bmod p) \quad \text { for } \quad(n, p)=1 .
$$

Particularly let us assume $n=g$, where $g$ denotes the primitive root of the prime number $p$; then we have

$$
g^{k} \equiv 1(\bmod p) \quad \text { where } \quad k<p-1
$$

contrary to the property of the primitive root. Therefore $S \geqslant p(p-1)$.

$2^{0} \quad a \geqslant 2$. I shall prove the impossibility of the conjecture

$$
S_{m}=p^{\alpha-1}(p-1) \quad \text { for }(n, p)=1 \text {. }
$$

Let us suppose the contrary. Admitting (7), we have

i. $e$.

$$
\left[n+p^{\alpha-1}(p-1)\right]^{n+p^{\alpha-1}(p-1)} \equiv n^{n}\left(\bmod p^{\alpha}\right)
$$

$$
n^{n}\left[\left(n^{p^{\alpha-1}(p-1)}-1\right)-n^{p^{\alpha-1}(p-1)} p^{\alpha-1}\right] \equiv 0\left(\bmod p^{\alpha}\right)
$$

Uring

$$
n^{p^{a-1}(p-1)}-1=n^{\psi\left(p^{u}\right)}-1 \equiv 0\left(\bmod p^{u}\right)
$$

we get

$$
n^{p^{a-1}(p-1)} p^{a-1} \equiv 0\left(\bmod p^{\alpha}\right)
$$

$$
((n, p)=1)
$$

which is impossible. It means that

$$
p^{\alpha} \mid S_{m} \quad \text { where } \quad S_{m} \mid p^{\alpha}(p-1) .
$$

We have therefore $S_{m}=p^{\alpha} k$.

I shall prove that $k=p-1$. Let us suppose $k<p-1$, then we have

$$
\left(n+S_{m}\right)^{n+S_{m}} \equiv n^{n}\left(\bmod p^{\alpha}\right)
$$

$$
n^{n+p^{\alpha} k} \equiv n^{n}\left(\bmod p^{\alpha}\right)
$$

it follows from (10) that

$$
n^{p a k}=n^{p^{\alpha-1}(p-1) k} n^{p^{\alpha-1} k} \equiv 1\left(\bmod p^{\alpha}\right) \quad((n, p)=1) .
$$

Let us take $n=g$ where $g$ denotes the primitive root of the number $p^{a}$. We have

i. e.

$$
n^{\varphi(m)}=n^{p^{a-1}(p-1)} \equiv 1\left(\bmod p^{\alpha}\right),
$$

$$
n^{p^{\alpha-1} k} \equiv 1\left(\bmod p_{\alpha}\right)
$$

$$
(k<p-1)
$$

contrary to the definition of the primitive root of a number. It means that $k \geqslant p-1$ and

(11)

$$
S_{m} \geqslant p^{\alpha}(p-1)
$$

from (6) and (11) we get immediately

$$
S_{m}=p^{\alpha}(p-1)
$$

Taking now $m=2 p^{a}, p \neq 2$ we have, as before,

$$
S_{m}=p^{\alpha}(p-1) \quad\left(S_{2 p^{a}}=S_{p^{a}}, p \neq 2\right) .
$$

Indeed, according to the theorem just proved we have

$$
n^{n} \cdot\left(n^{p^{p^{\alpha}(p-1)}}-1\right) \equiv 0\left(\bmod p^{\alpha}\right) \quad((n, p)=1) .
$$

If $n \equiv 0(\bmod 2)$, the relation (12) is obvious.

If $n \equiv 1(\bmod 2), n^{p^{\alpha}(p-1)}-1 \equiv 0(\bmod 2)$ and $\left(\bmod p^{\alpha}\right)$ if $(n, p) \neq 1$ i. e. $p \mid n$ the relation (12) is valid (see later) at any rate for $n \geqslant \alpha$.

Thus we have, beginning from some $n$,

$$
n^{n+p^{a}(p-1)} \equiv n^{n}\left(\bmod 2 p^{a}\right)
$$


For instance

$$
\begin{gathered}
m=2^{\prime a}, \quad S_{m}=2^{a}=m, \\
m=10, \quad S_{m}=5 \cdot(5-1)=20 .
\end{gathered}
$$

Now I shall discuss when the period is proper and when it is mixed in the cases $m=p^{a}$ and $m=2 p^{a}, p \neq 2$.

Let us take first

$$
m=p^{a} .
$$

When $p \geqslant a$, the period is proper. Indeed, for $(n, m)=1$ the period begins from $n=1$.

Let us suppose $(n, m) \neq 1$ i. e. $(n, m)=p^{\beta} ; 1 \leqslant \beta \leqslant \alpha \leqslant p ;$ i. e. $n=p^{\beta}$.t. We have

$$
\left(n+S_{m}\right)^{n+S_{m}} \equiv n^{n}=\left(p^{\beta} t\right)^{p^{\beta} t} \equiv 0\left(\bmod p^{a}\right)
$$

for every natural $t$ and $\beta$.

If $p<a$; the period is necessarily mixed because

$$
\left(p+S_{m}\right)^{p+S_{m}} \equiv p^{p+S_{m}} \neq p^{p}\left(\bmod p^{\alpha}\right),
$$

it is sufficient but not necessary to begin the period from $n=a$.

We shall find the necessary and sufficient condition as follows. It is evident that we have to discuss only the ease $(n, p) \neq 1$. i. e. $n=p^{i}$, $1 \leqslant \beta \leqslant \alpha$. We seek the least natural number $k$ such that

i. e.

$$
(k p)^{k p} \equiv 0\left(\bmod p^{\alpha}\right)
$$

$$
k p \geqslant a ; \quad(k-1) p<a, \quad-\frac{a}{p}-1<-k \leqslant-\frac{a}{p}, \quad k=-\mathrm{E}\left(-\frac{a}{p}\right) .
$$

Denoting by $n_{0}=n_{a p}$ the natural number from which the period begins we get immediately

$$
n_{0}=n_{\alpha p}=(k-1) p+1=1-p\left[1+\mathrm{E}\left(-\frac{a}{p}\right)\right] .
$$

Particularly if $a \leqslant p$, we obtain from (14) the result given above. I omit the case $m=2 p^{a}$ which leads to quite the same result $e . g$. $p=7, \alpha=15, n_{0}=\alpha=15 ; \quad p=7, \alpha=14, n_{0}=8 ; \quad p=2, \alpha=3, \quad n_{0}=3$ ete.

Before proceeding to the general case I shall give two quite evident relations:

$1^{0}$ If $m_{1} \mid m$, then $S_{m_{1}} \leqslant S_{m}$. Indeed, denoting $u_{k}=k^{k}$, if $u_{k+S_{m}} \equiv u_{k}$ $(\bmod m)$, then, all the more, $u_{k+S_{m}} \equiv u_{k}\left(\bmod m_{1}\right)$.
$2^{0}$ If $S_{1}$ and $S_{2}$ are two different periods of the rests of numbers mod $m$ then $\left(S_{1}, S_{2}\right)=$ (g. c. d. of the numbers $S_{1}$ and $\left.S_{2}\right)$ forms also the period $\bmod m$. Let us take $n_{n} \geqslant n_{0}$. According to our supposition

and

$$
u_{n+S_{1}} \equiv u_{n}(\bmod m), \quad u_{n+S_{2}} \equiv u_{n}(\bmod m)
$$

$u_{n+k S_{1}+t S_{2}} \equiv u_{n}(\bmod m) \quad\left(k, t\right.$ integers $\left.n_{t}+k S_{1}+t S_{2} \geqslant n_{0}\right) ;$

but the equation (indefinite) $k S_{1}+t S_{2}=\left(S_{1}, S_{2}\right)$ has an infinity of solutions $(k, t)$, so that we may write

$$
\left[n+\left(S_{1}, S_{2}\right)\right]^{n+\left(S_{1}, S_{2}\right)} \equiv n^{n}(\bmod m)
$$

for every sufficiently great $n$.

Remark 1. Considering $S_{m} \geqslant 2(m \geqslant 2)$, the relation $\left(S_{1}, S_{2}\right)=1$ is impossible; $i$.e. there do not exist two relatively prime periods.

Remark 2. It is obvious that the last two assertions may be applied to the rests of terms of every periodical sequence $\bmod m$.

Let us proceed to the general case $m=\prod_{l=1}^{k} p_{l}^{a_{l}}$.

THEOREM. Denoting as before by the symbol $\left\{a_{i}\right\}$ the l. o. m. of the numbers $a_{1}, a_{2}, \ldots, a_{k}$ we have

Proof.

$$
S_{m}=\left\{p_{i} \varphi\left(p_{i}^{a_{i}}\right)\right\}=\left\{m ; p_{i}-1\right\} \quad(i=1,2, \ldots, k) .
$$

$\left[n+\left\{m ; p_{i}-1\right\}\right]^{n+\left\{m ; p_{i}-1\right\}} \equiv n^{n}\left(\bmod p_{i}^{a_{i}}\right), \quad i=1,2, \ldots, k, \quad$ for $\quad n \geqslant n_{\alpha_{i} p_{i}}$ and since all the natural numbers $p_{1}^{a_{1}}, p_{2}^{a_{2}}, \ldots, p_{k}^{a_{k}}$ are relatively prime, we conclude that

(16) $\quad\left[n+\left\{m ; p_{i}-1\right\}\right]^{n+\left\{m ; p_{i}-1\right\}} \equiv n^{n}(\bmod m), \quad$ for $n \geqslant \max \left(n_{a_{i} p_{i}}\right)$

it means that

Let us suppose that

$$
S_{m} \leqslant\left\{p_{i} \varphi\left(p_{i}^{a_{i}}\right)\right\}
$$

$$
s_{m}<\left\{p_{i} \varphi\left(p_{i}^{a_{i}}\right)\right\}
$$

then we have $p_{i} \varphi\left(p_{i}^{\alpha_{i}}\right) \nmid S_{m}$ at least for some $i=i_{0}$, and, according to the second assertion mentioned above, the number

$$
L=\left(S_{m} ; p_{i_{0}}^{a_{i_{0}}}\left(p_{i_{0}}-1\right)\right)<p_{i_{0}}^{\alpha_{i_{0}}}\left(p_{i_{0}}-1\right)=S p_{i_{0}}^{a_{i_{0}}}
$$

would be a period mod $p_{i_{0}}^{a_{i_{0}}}$ which is impossible. Therefore

$$
S_{m}=\left\{p_{i} p\left(p_{i}^{a_{i}}\right)\right\}
$$


It is interesting to answer the question when $m=S_{m}$. The necessary condition is $m \equiv 0(\bmod 2), e . g$.

$$
m=2^{\alpha}, \quad m=2^{\alpha} \cdot 3^{\beta}, \quad m=2^{\alpha} 5^{\beta} \quad(\alpha \geqslant 2), \quad m=2^{\alpha} 3^{\beta} 7^{\gamma}
$$

for all these cases $S_{m}=m$.

It means that the number

$$
\varphi(m) \prod_{l=1}^{k} p_{l}
$$

given on the first page as the period, may be divided by

$$
2^{\alpha_{1}+\sum_{l=1}^{k} \nu_{l}-\max \left(\gamma_{i} ; \alpha_{i}\right)} \quad \text { when } \quad p_{1}=2
$$

and by

$$
2^{\sum_{i=1}^{k} \gamma_{t}-\max \gamma_{i}} \quad \text { when } \quad p_{1} \geqslant 3 \quad(i=1,2, \ldots, k),
$$

where

$$
p_{i}-1=2^{\gamma_{i}} c_{i}, \quad o_{i} \neq 0(\bmod 2) . \quad(i=1,2, \ldots, k),
$$

and possibly by other (necessarily odd) divisors of the numbers $o_{i}$.

The period is proper if $p_{i} \geqslant a_{i}, i=1,2, \ldots, k$, and mixed if this is not the case. If we denote

$$
n_{\alpha_{i}, p_{i}}=1-p_{i}\left[1+\mathrm{E}\left(-\frac{a_{i}}{p_{i}}\right)\right]
$$

the period begins in both cases from $n=\max \left(n_{a_{i} p_{i}}\right), i=1,2, \ldots, k$.

\section{Problème du mouvement stationnaire dans une couche gazeuse rayonnante}

par W. PogorzelskI (Warszawa)

Introduction. L'étude de l'équilibre ou du mouvement dans un grand milieu gazeux, comme l'atmosphère d'une planète ou d'une étoile, doit tenir compte du rayonnement intérieur en négligeant l'influence de la conductibilité.

K. Schwarzschild [4] le premier et ensuite R. Emden [2] ont étudié l'équilibre d'une couche gazeuse en tenant compte du rayonnement mais sous la supposition inexacte des courants d'énergie dans deux directions seulement.

C. Białobrzeski [1] a étudié le premier l'équilibre d'une sphère gazeuse en tenant compte de la pression de radiation.

W. Pogorzelski [3] a étudié l'équilibre d'une couche gazeuse en tenant compte $d u$ rayonnement polychromatique dans toutes les directions. Le problème conduit à un système d'équations intégrales non linéaires, assez compliquées; l'auteur a démontré l'existence de la solution si l'épaisseur de la couche est suffisamment petite.

Dans ce travail nous démontrerons l'existence d'un état stationnaire du mouvement dans une couche gazeuse en tenant compte du rayonnement polychromatique dans toutes les directions. Le problème consiste en l'étude d'un système d'équations intégrales non linéaires.

Les grandeurs et les équations fondamentales. Soit au point intérieur $M$ d'un milieu gazeux un élément de surface $\boldsymbol{d} \sigma \sigma$ avec la normale $M n$. On admet que la quantité d'énergie, qui passe par l'élément $\boldsymbol{d \sigma}$ dans le temps $d t$ grâce au rayonnement de longueur d'onde dans l'intervalle $(\lambda, \lambda+d \lambda)$ dans la direction $M R$ d'un angle solide $d \omega$, a pour valeur principale le produit

$$
X \cos \Theta d \sigma d \lambda d t d \omega,
$$

$\Theta$ étant l'angle que fait la direction $M R$ avec la normale $M n$. La grandeur $X$ s'appelle l'intensité de rayonnement de longueur d'onde $\lambda$, au point $M$ et dans la direction $M R$.

Annales Polonici Mathematici I. 